\title{
Untersuchung der induzierten Emission von gepulsten Argon- und Xenon-Ionenlasern im sichtbaren und ultravioletten Bereich
}

\author{
W. Demtröder und E. Elendt \\ Physikalisches Institut der Universität Freiburg i. Br. \\ (Z. Naturforschg. 21 a, 2047-2054 [1966] ; eingegangen am 24. August 1966)
}

\begin{abstract}
Time behaviour, threshold currents and power output of the most intensive induced transitions are investigated in pulsed argon- and xenon-ionlasers in the visible and ultraviolet region. In the $7 \mathrm{~mm}$ diameter tube transitions of higher ionized atoms are favoured compared to smaller diameter tubes. Pressure dependence of threshold currents and curent dependence of induced peak power are measured. By observing discharge current, spontaneous sidelight and laser output at the same time, information is obtained about possible excitation mechanisms for various transitions.
\end{abstract}

Im sichtbaren und ultravioletten Spektralbereich sind bei Ar- und Xe-Ionenlasern inzwischen über 100 verschiedene induzierte Übergänge gefunden worden ${ }^{1-3}$. Die meisten dieser Linien wurden nur photographisch registriert, um ihre genaue Wellenlänge und die zugehörigen Terme des ein- oder mehrfach geladenen Ions zu bestimmen. Sehr eingehend sind diejenigen sichtbaren induzierten Übergänge im ArII in der Literatur behandelt worden, die auch in kontinuierlich betriebenen Lasern angeregt werden können, und die Anregungsmechanismen sowie beobachtete Sättigungserscheinungen sind für diese Übergänge wenigstens teilweise geklärt ${ }^{4-9}$.

In dieser Arbeit sollen die intensivsten sichtbaren und ultravioletten Linien in gepulsten Ar- und XeIonenlasern untersucht werden. Aus dem zeitlichen Verhalten der induzierten Emission bei verschiedenen Gasdrucken, Entladungsströmen und Feldstärken kann man Aufschlüsse erhalten über optimale Anregungsbedingungen, um daraus, vor allem für die ultravioletten Linien, maximale Ausgangsleistungen oder -energien zu erreichen. Durch gleichzeitige Messung des Entladungsstroms, der spontanen und induzierten Emission gewinnt man außerdem Information über die verschiedenen Anregungsmechanismen, die zur Inversion führen können.

1 W. B. Bridges u. A. N. Chester, IEEE Quant. Electr. 1, 66 [1965] ; Appl. Opt. 4, 573 [1965].

2 W. B. BRIDGes, Internat. Conf. on Quant. Electr. 1966, in IEEE, Quant. Electr. [1966].

3 P. K. Cheo u. H. G. Cooper, J. Appl. Phys. 36, 1862 [1965].

4 J. M. Hammer u. C. P. Wen, Appl. Phys. Letters 7, 159 [1965].

5 P. K. Cheo u. H. G. Cooper, Appl. Phys. Letters 6, 177 [1965].

\section{Experimenteller Aufbau}

Die Anregung der induzierten Emission erfolgte durch eine stromstarke Kondensatorentladung. Als Entladungsrohr wurde eine $1 \mathrm{~m}$ lange Quarzröhre mit $7 \mathrm{~mm}$ Innendurchmesser, BREwSTER-Endfenstern und eingeschmolzenen kalten Wolframelektroden verwendet. Die Kondensatorkapazität konnte zwischen $1 \mu \mathrm{F}$ und $40 \mu \mathrm{F}$ variiert werden bei Spannungen bis zu $20 \mathrm{kV}$. Die Entladung wurde gezündet durch einen um die ganze Länge des Laserrohres gewickelten Draht, auf den durch eine Thyratronentladung über einen Hochspannungstransformator ein $20 \mathrm{kV}$-Puls gegeben wurde. Durch verschiedene Widerstände im Entladungskreis konnte die Stromstärke bei gleicher Spannung variiert werden und durch entsprechende Drosseln der Stromanstieg. Der zeitliche Stromverlauf wurde aus dem Spannungsabfall an einem induktionsarmen Widerstand vom oberen Strahl eines Tektronix-Zweistrahloszillographen 551 gemessen, während gleichzeitig der untere Strahl die induzierte Emisison und die spontane Emission des oberen Laserniveaus aufzeichnete. Die Pulsfolgefrequenz konnte von $0-20 \mathrm{~Hz}$ variiert werden, die meisten Untersuchungen wurden aber bei niedrigen Frequenzen (etwa $0,1 \mathrm{~Hz}$ ) gemacht, weil sich sonst im Verlauf einer Meßreihe durch Erwärmung der Entladungsröhre und durch Pumpprozesse infolge der starken Ionenströme (die Entladungsstromstärken betrugen bis $6000 \mathrm{~A}$ ) die Druckverhältnisse im Laserrohr in unkontrollierbarer Weise ändern. Der Fülldruck wurde mit einem $\mathrm{H}$ e r a e u s - Autovac-Thermotron gemessen, das für die einzelnen Gase geeicht war. Alle angegebenen Drucke beziehen sich auf Zimmertemperatur.

6 E. I. Gordon u. E. F. Labuda, Appl. Phys. Letters 4, 178 [1964].

7 E. I. Gordon u. E. F. Labuda, Conf. on Quant. Electr. 1965, edited by McGraw-Hill [1966], S. 380 ff.

8 W. Demtröder, Phys. Letters 22, 436 [1966].

9 E. A. Ballik, W. R. Bennet jr. u. G. N. Mercer, Appl. Phys. Letters 8, 214 [1966]. 
Der optische Resonator bestand aus einem sphärischen Aluminiumspiegel $(R=140 \mathrm{~cm}$, aufgedampfter Alu-Belag Alfex U, Fa. B alzers) mit einem Reflexionsvermögen von etwa $90 \%$ für $\lambda>220 \mathrm{~m} \mu$ und einem totalreflektierenden $90^{\circ}$-Suprasilprisma, dessen Basisfläche vergütet war. Für die sichtbaren Linien wurden wahlweise auch dielektrische Breitbandspiegel verwendet (Reflexionsvermögen größer als 98\% zwischen 440 und $640 \mathrm{~m} \mu$; „Kaltlichtspiegel“, Fa. B a l z e r s). Ausgekoppelt wurde der Reflexionsanteil an einem Brewster-Fenster, der infolge geringer Abweichung vom BREwSTER-Winkel für die verschiedenen Wellenlängen $0,2-0,5 \%$ der im Resonator gespeicherten induzierten Leistung betrug.

Über ein Spiegel- und Linsensystem wurde diese ausgekoppelte induzierte Emision auf den Eintrittsspalt eines Quarzprismen-Spektrographen abgebildet und am Ausgang mit einer Photoplatte zur Wellenlängenbestimmung oder mit einem Photomultiplier zur Untersuchung der Leistung und des zeitlichen Emissionsverlaufs registriert. Dabei mußten die intensiven Linien durch entsprechende Graufilter vor dem Spektrographenspalt (oder dünne Glasscheiben im UV-Bereich) bis zu $10^{4}$-fach geschwächt werden, da sonst die Photokathode übersteuert wurde. Da der Eingangsspalt aus Gründen der Linientrennung im sichtbaren Gebiet nur $0,05 \mathrm{~mm}$ breit und damit etwas schmaler als der kreisförmige Fokus der zu untersuchenden Strahlung war, wurde durch den Spektrographen aus der Gesamtstrahlung ein Streifen ausgeschnitten, in dem hauptsächlich die induzierte Emision aus der Rohrmitte gemessen wurde, so daß z. B. bei Untersuchungen der Sättigungserscheinung überwiegend Vorgänge in der Rohrmitte erfaßt werden ${ }^{5}$.

Die spontane Emission wurde von der Seite des Laserrohres, senkrecht zur Rohrachse, mit einem zweiten Photomultiplier beobachtet, wobei die Wellenlängenselektion durch einen Le is s-Monochromator erfolgte.

\section{Meßergebnisse}

\section{Argon, sichtbare Linien}

In Abb. 1 ist für den Übergang des ArII $\left(3 \mathrm{~s}^{2} 3 \mathrm{p}^{4}\left({ }^{3} \mathrm{P}\right) 4 \mathrm{p} \rightarrow 4 \mathrm{~s}\right)$ bei $\lambda=4878,9 \AA$ der zeitliche Verlauf des Entladungsstromes - I, der Laserausgangsleistung $L$ und der spontanen Emission $S$ vom oberen Niveau bei verschiedenen Gasdrucken aufgenommen worden unter sonst gleichen Bedingungen. Bei 60 mTorr, dem höchsten Druck, bei dem induzierte Emission auftrat, erscheint ein schwacher Laserpuls erst auf der abfallenden Flanke des Strompulses. Bei kleineren Drucken erhält man schon während des Stromanstieges induzierte Emission, die aber im Be-

10 F. A. Horrigan, S. H. Koozekanani u. R. A. Pananen, Appl. Phys. Letters 6, 41 [1965]. reich mittlerer Drucke bei größerem Strom wieder völlig aufhört und erst erneut einsetzt, wenn der Strom sein Maximum überschritten hat. Der Abbau der Inversion bei hohen Strömen wird dadurch verursacht, daß bei zu großer Ionendichte die Resonanzfluoreszenz vom unteren Niveau zum Ionengrundzustand $(\lambda=720 \AA)$ fast völlig absorbiert wird ${ }^{5}$. Dadurch wird die Lebensdauer dieses Niveaus um einen Betrag verlängert, der proportional zur Ionendichte ist, und der den spontanen Wert von $1,8 \mathrm{~ns}^{10}$ um Größenordnungen übertreffen kann. Sobald diese effektive Lebensdauer größer als die des oberen Niveaus wird (10,0 ns ${ }^{11}$, kann bei gleicher Anregungsrate für beide Niveaus keine Inversion mehr aufrechterhalten werden. Da das untere Niveau optisch mit dem Ionengrundzustand verbunden ist, das obere aber nicht, wird bei großen Drucken, bei denen die Anregung beider Niveaus in zwei Schritten über den Grundzustand oder metastabile Niveaus des Ions erfolgt, die Anregungsrate für das untere Niveau größer sein als für das obere. Die Inversion wird deshalb schon früher zerstört, und es kann statt Verstärkung der induzierten Emission starke Absorption auftreten ${ }^{7}$. Wegen des großen Rohrdurchmessers ist diese Resonanzabsorption bei unserer Anordnung besonders wirksam. Deshalb liegen die hier gemessenen Maximalstromdichten niedriger als bei Experimenten mit kleinerem Rohrquerschnitt ${ }^{3}$. Während der Entladung ändern sich die Druckverhältnisse im Rohr durch Pumpprozesse, außerdem erwärmt sich das Gas, so daß durch steigende Doppler-Verbreiterung die Resonanzabsorption bei $\lambda=720 \AA$ und damit die Besetzungszahl des unteren Niveaus sinkt. Dadurch kann am Ende des Strompulses wieder induzierte Emission möglich sein (Abb. 1 a). Mit sinkendem Druck wird bei gleicher Stromstärke die Ionendichte kleiner ${ }^{12}$, deshalb tritt der Inversionsabbau durch Resonanzabsorption erst bei größeren Stromdichten auf (Abb. $1 \mathrm{~b}-\mathrm{e}$ ). Bei hohen Drucken wächst die spontane Intensität während des Stromanstieges etwa proportional zu $I^{2}(\mathrm{Abb} .1 \mathrm{a})$, mit sinkendem Druck geht diese quadratische Abhängigkeit immer mehr in eine lineare über und die spontane Emission fällt nach Erreichen eines deutlichen Maximums etwas ab, um dann erneut anzusteigen (Abb. 1d). Außerdem wird die Verzögerungszeit der induzierten Emission gegen den Strombeginn

11 J.Bakos, J. Scigeti u. L.VArga, Phys. Letters 20, 503 [1966]. 12 P. K. Cheo u. H. G. Cooper, Appl. Phys. Letters 7, 202 [1965]. 


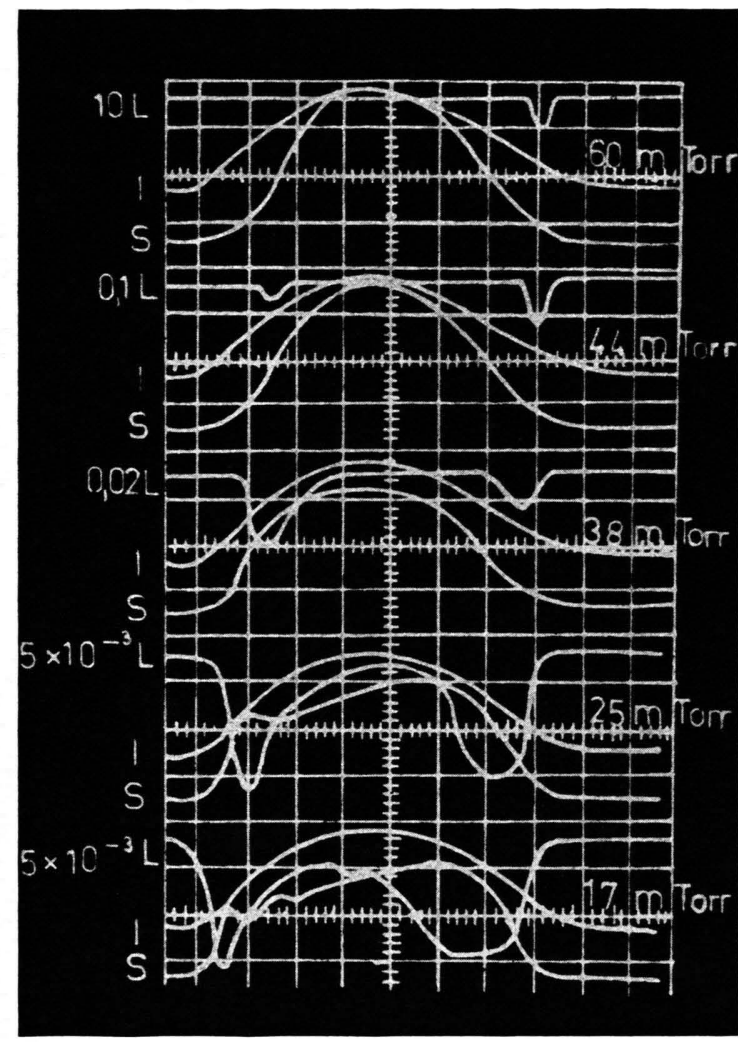

Abb. 1. Zeitlicher Verlauf von Entladungsstrom I, Laserausgangsleistung $L$ und spontaner Emission $S$ für verschiedene Einfülldrucke. Zeit: $100 \mu \mathrm{s} / \mathrm{cm}$, Strom: $250 \mathrm{~A} / \mathrm{cm} ; 40 \mu \mathrm{F}$, 10 mHenry.

immer kleiner und der erste Laserpuls wird höher als der zweite. Die Schwellwertstromdichte für den Einsatz des ersten Pulses liegt bei hohen Drucken höher als für das Ende des zweiten Pulses, mit ab-

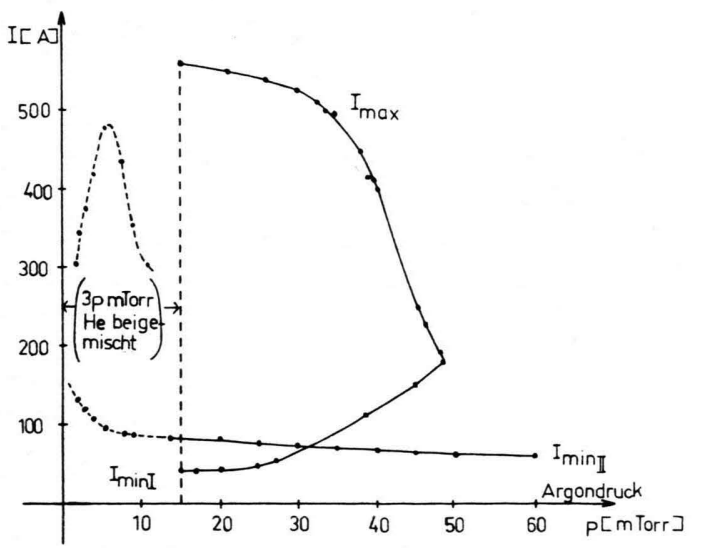

Abb. 2. Druckabhängigkeit der oberen Stromgrenze $I_{\max }$ und des minimalen Schwellwertstromes $I_{\min }$ I für direkte Anregung und $I_{\min }$, II für den Zweistufenprozeß. nehmendem Druck wird diese Differenz kleiner und kehrt ihr Vorzeichen um bei kleinen Drucken.

Alle diese Beobachtungen weisen darauf hin, daß mit sinkendem Druck zu Beginn der Entladung die direkte Anregung des oberen Laserniveaus durch Elektronenstoß vom neutralen Grundzustand aus neben dem Zweistufenprozeß wirksam wird und bei niedrigen Drucken und hohen Feldstärken die dominierende Rolle spielt ${ }^{8}$. In Abb. 2 ist die Druckabhängigkeit des "Quenching-Stromes" $I_{\max }$ sowie des minimalen Schwellwertstromes $I_{\min }$ für beide Anregungsprozesse aufgetragen. Die Stromstärke, bei der der zweite Laserpuls aufhört, ergibt im wesentlichen den Schwellwertstrom für den Zweistufenprozeß, weil die direkte Anregung wegen der weitgehenden Ionisierung des Neutralgases am Ende des Strompulses keine Rolle mehr spielt. Bei kleinen Drucken ist die Stromstärke bei Beginn des ersten Laserpulses ein Maß für den Schwellwert, der zur direkten Anregung gehört und der kleiner ist als für den Zweistufenprozeß. Mit wachsendem Druck verliert die direkte Anregung an Bedeutung, der Schwellwert konvergiert gegen den der Zweistufenanregung. Das stärkere Anwachsen von $I_{\min , \mathrm{I}}$ in Abb. 2 mit steigendem Druck liegt vor allem an der zunehmenden Verzögerungszeit zwischen Strompuls und Laserpuls, die von $0,5 \mu \mathrm{s}$ bei kleinen Drucken bis zu $500 \mu \mathrm{s}$ bei großen Drucken wachsen kann. Sobald diese Zeit größer als die Stromanstiegszeit wird, erscheint nur noch ein Laserpuls auf der abfallenden Strompulsflanke. Der Schnittpunkt zwischen $I_{\max }$ und $I_{\min , \mathrm{I}}$ verschiebt sich deshalb mit wachsender Strompulsdauer zu höheren Drucken, und im Dauerbetrieb werden alle drei Kurven vermutlich sich in einem Punkte schneiden, wie aus einer Extrapolation von Meßwerten bei verschiedenen Entladungsdauern zu entnehmen ist.

Das hier geschilderte zeitliche Verhalten der induzierten Emission ist typisch für alle Linien, die auch kontinuierlich angeregt werden können: Abgesehen von dem zusätzlichen direkten Anregungsmechanismus, der nur so lange wirksam ist, wie die Ionendichte nicht zu groß wird, kann die Inversion dauernd aufrechterhalten werden, so lange der Strom innerhalb gewisser Grenzen bleibt. Diese Grenzen nähern sich mit steigendem Druck einander und bestimmen durch ihren Schnittpunkt den Maximaldruck, bei dem überhaupt Inversion erreicht werden kann. Die untere Druckgrenze ist bei reiner Argonfüllung apparativ bedingt, weil bei Drucken unter 
10 mTorr die Gasentladung bei Spannungen unter $5 \mathrm{kV}$ und bei Verwendung von kalten Elektroden nicht mehr zuverlässig zündet. Durch Beimischen von Helium als Puffergas kann diese Schwierigkeit umgangen werden. Die Werte, die aus Entladungen in einem $\mathrm{Ar}-\mathrm{He}-\mathrm{Gemisch}(\mathrm{Ar}: \mathrm{He}=1: 3$ ) erhalten wurden, sind in Abb. 2 gestrichelt eingezeichnet. Der Maximalstrom zeigt hier ein anderes Druckverhalten als im reinen Argon. Der Argondruck ist so niedrig, daß Resonanzabsorption keine entscheidende Rolle mehr spielen kann. Vermutlich wird die Inversion bei großen Strömen durch deaktivierende Stöße mit He-Ionen abgebaut. Da der Schwellwertstrom für den Zweistufenprozeß mit sinkendem Druck zunimmt, wird man im Dauerbetrieb, bei dem man nicht so hoch oberhalb der Schwelle arbeitet, maximale Ausgangsleistung bei höheren Drucken erreichen, als im Pulsbetrieb, bei dem man bald in das Gebiet des "Quenching-Stromes" kommt. Eine weitere Ursache für die größeren optimalen Drucke im Dauerbetrieb ist allerdings auch die höhere Temperatur im Entladungsrohr gegenüber der bei Pulsbetrieb ${ }^{7}$.

\section{Argon, ultraviolette Linien}

Die ultraviolette induzierte Emission des Ar III bei $\lambda=3511 \AA$ und des Ar IV bei $\lambda=2913 \AA$ zeigt ein zeitliches Verhalten, das der direkten Anregung der Ar II-Linie ähnlich ist. In Abb. 3 sind zum Vergleich alle drei induzierten Übergänge gezeigt. Während aber für $\lambda=4880 \AA$ die Inversion nach einem kurzen, intensiven Laserpuls erneut wieder aufgebaut wird durch den Zweistufenprozeß, der induzierte Emission ermöglicht, solange der Entladungsstrom oberhalb des Schwellwertes bleibt, wird für die ultravioletten Übergänge Inversion nur zu Beginn des Strompulses während des Stromanstieges erreicht und man erhält lediglich einen kurzen Laserpuls, der für $\lambda=3511 \AA$ etwa $1-2 \mu \mathrm{s}$, für $\lambda=2913 \AA$ etwa $0,2-0,5 \mu$ s lang ist. Aus dem spontanen Emissionsverlauf sieht man, daß die Besetzung des oberen Niveaus bis zum Strommaximum steil ansteigt, dann rasch und später langsam abfällt. Diesen Verlauf erhält man auch ohne Resonatorspiegel, so daß der Besetzungsabbau des oberen Niveaus nicht durch induzierte Emission geschieht.

Mit steigender Spannung und sinkendem Druck werden die Pulse immer schmaler und bis 20 mTorr auch höher. Bei tieferen Drucken fällt die induzierte

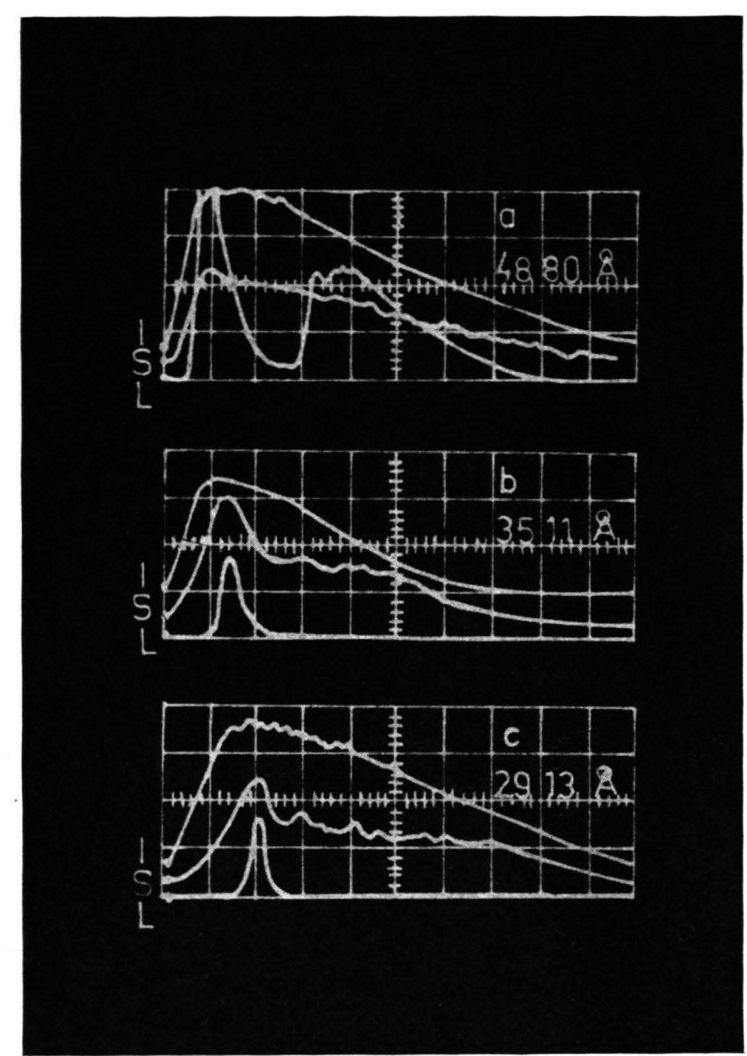

Abb. 3. Vergleich des zeitlichen Verlaufs der induzierten und spontanen Emission für Übergänge im Ar II (a), Ar III (b), beide $2 \mu \mathrm{s} / \mathrm{cm}, \operatorname{Ar}$ IV (c), $1 \mu \mathrm{s} / \mathrm{cm}$.

Leistung dann steil ab. Verlangsamt man den Stromanstieg durch Einschalten einer variablen Induktivität in den Entladekreis, so sinkt bei gleichem Maximalstrom die Spitzenleistung schnell ab, d. h. für den Anregungsmechanismus ist nicht nur ein Minimalstrom, sondern auch eine möglichst kurze Stromanstiegszeit notwendig.

Die Entladungsdauer hat auf die Pulshöhe und Dauer der induzierten Emission keinen Einfluß, solange sie größer als die Laserpulsdauer ist, d. h. eine Erhöhung der Kapazität bringt keine Steigerung der induzierten Ausgangsleistung im Gegensatz zu den sichtbaren Linien der Ar II.

Im Druckbereich um $30 \mathrm{mTorr}$ erscheint allerdings bei $3511 \AA$ noch ein zweiter, sehr schwacher Puls, dessen Amplitude etwa $10^{3}$-mal kleiner ist als die des ersten, dessen Breite aber mit steigender Entladungsdauer zunimmt, der also auf einen möglichen kontinuierlichen Betrieb auf dieser Linie schließen läßt. Der Schwellwertstrom für diesen Puls ist etwa 
$200 \AA$; dabei ist die Verstärkung der induzierten Emission größer als $12 \%$ pro Meter ${ }^{*}$.

Die Ausgangsenergie ist bei $\lambda=4880 \AA$ wegen der größeren Pulsdauer größer als bei den ultravioletten Linien. Bei Verwendung kleinerer Kondensatoren und damit möglichen höheren Folgefrequenzen kann man aber z. B. bei $\lambda=3511 \AA$ etwa gleich große mittlere Leistungen erzielen, wie bei $4880 \AA$. Die zur Anregung nötigen Stromdichten sind um so höher, je höher der Ionisationsgrad ist. In Abb. 4 ist für die drei Linien die induzierte Leistung als Funktion der Maximalstromstärke während der Entladung aufgetragen. Bei konstanter Stromstärke steigt die Leistung mit steigender Spannung bei dem einfach ionisierten Übergang nur wenig, bei $\lambda=$ $3511 \AA$ merklich und bei $\lambda=2913 \AA$ sehr stark.

Der Druckbereich, in dem induzierte Emission möglich ist, wird mit zunehmendem Ionisationsgrad enger und liegt für die höher ionisierten Übergänge etwas höher, als für die direkte Anregung der einfach ionisierten Linie. Bei größerer Entladungsdauer weitet sich aber für die letztere der Druckbereich infolge des zusätzlichen Anregungsmechanismus aus und optimale Anregung erfolgt bei höheren Drukken. In Abb. 5 ist diese Druckabhängigkeit der jeweils erreichten Maximalintensität aufgetragen. Für

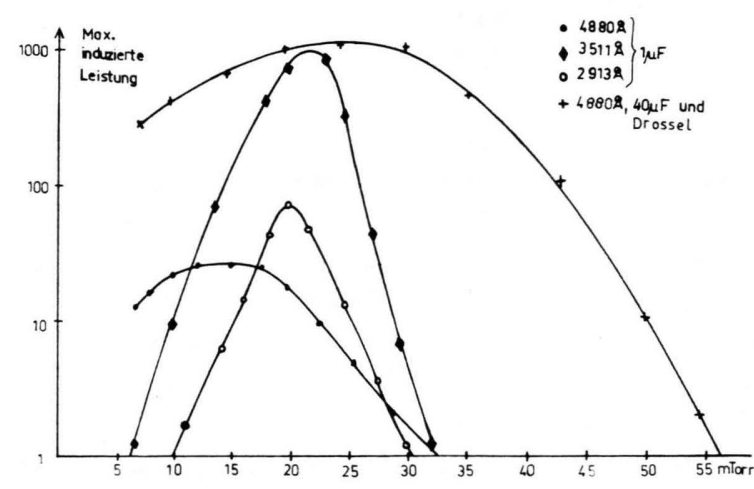

Abb. 5. Maximal erreichbare induzierte Emission der drei Argonlinien als Funktion des Einfülldruckes.

alle Linien wurden als Resonator Aluminiumspiegel und Quarzprisma verwendet. Bei den ultravioletten Linien hängt die maximal erreichbare Leistung vom Verhältnis $E / p$ ab, d. h. bei größeren Drucken braucht man höhere Feldstärken. Mit sinkendem Druck sinkt zwar auch der Schwellwertstrom, bei dem induzierte Emission auftritt, aber bei Drucken unter $20 \mathrm{mT}$ Torr erreicht man mit steigendem Entladungsstrom bald einen zum Druck proportionalen Sättigungswert der induzierten Emission, so daß die maximal erreichbare Leistung wieder kleiner wird.

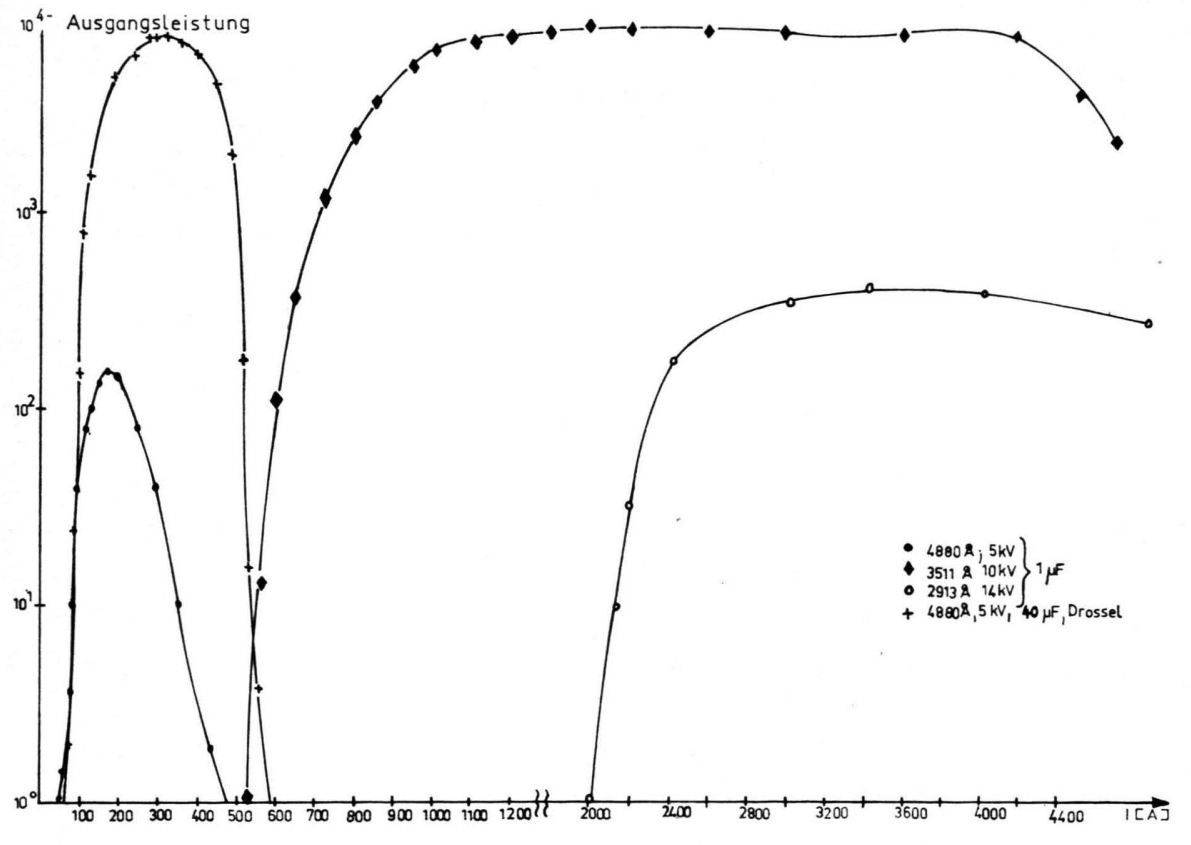

Abb. 4. Stromabhängigkeit der induzierten Ausgangsleistung (relative Einheiten) für die drei Argonlinien bei jeweils optimalem Druck.

* Zusatz bei der Korrektur: Inzwischen ist auf dieser Linie kontinuierliche induzierte Emission erreicht worden (R. PaAnanen, Appl. Phys. Letters 9, 34 [1966]). 
Ein ähnliches Sättigungsverhalten zeigt auch die spontane Emission. Das weist darauf hin, daß für die Begrenzung der erreichbaren Inversion nicht, wie bei den Ar II-Übergängen die verlängerte Lebensdauer des unteren Niveaus durch Reabsorption verantwortlich ist, sondern daß bei den Sättigungsstromwerten die Ionenkonzentration des untersuchten Überganges ihren Maximalwert erreicht hat und bereits größer als die Neutralgasdichte ist, so daß bei weiterer Stromzunahme höhere Ionisationsstufen angeregt werden auf Kosten der niedrigeren.

Beim Ar III geschieht die Anregung des oberen Niveaus wahrscheinlich durch Elektronenstoß von Zuständen des Ar II aus, die mit diesem Niveau optisch verbunden sind, denn im Strombereich um den Schwellwert der induzierten Emission steigt die spontane Intensität bei diesen Übergängen proportional zum Quadrat des Entladungsstromes. Bei höheren Strömen kann man dann allerdings keine so einfache Abhängigkeit mehr finden, weil die Verminderung der Neutralgasdichte eine Rolle spielt und Kaskaden von höheren Zuständen aus den zeitlichen Verlauf der spontanen Emission verändern.

In Tab. 1 sind für die untersuchten Argonlinien die Schwellwertströme (gemessen als Spitzenwert des Entladungsstromes, bei dem gerade noch induzierte Emission auftritt) und die maximal erreichte ausgekoppelte Leistung (Auskopplungsgrad etwa $0,5 \%$ ) zusammengestellt. Die Termzuordnung ist Tabellen von BRIDGES ${ }^{1}$ entnommen.

\begin{tabular}{|c|c|c|c|}
\hline $\begin{array}{c}\text { Wellen- } \\
\text { länge } \\
(\AA)\end{array}$ & $\begin{array}{c}\text { Ionisationsgrad } \\
\text { und Utbergang } \\
\text { (nach BRIDGES }\end{array}$ & $\begin{array}{c}\text { Minimaler } \\
\text { Schwell- } \\
\text { wert- } \\
\text { strom } \\
\text { (Ampere) }\end{array}$ & $\begin{array}{c}\text { Ausge- } \\
\text { koppelte } \\
\text { Spitzen- } \\
\text { leistung } \\
\text { (Watt) }\end{array}$ \\
\hline 5145 & II; $4 \mathrm{p}^{4} \mathrm{D}_{5 / 2}^{0} \rightarrow 4 \mathrm{~s}^{2} \mathrm{P}_{3 / 2}$ & 130 & 0,02 \\
4965 & II; $4 \mathrm{p}^{2} \mathrm{D}_{3 / 2}^{0} \rightarrow 4 \mathrm{~s}^{2} \mathrm{P}_{1 / 2}$ & 110 & 0,1 \\
4880 & II; $4 \mathrm{p}^{2} \mathrm{D}_{5 / 2}^{0} \rightarrow 4 \mathrm{~s}^{2} \mathbf{P}_{3 / 2}$ & 45 & 0,2 \\
4765 & II; $4 \mathrm{p}^{2} \mathrm{P}_{3 / 2}^{0} \rightarrow 4 \mathrm{~s}^{2} \mathrm{P}_{1 / 2}$ & 100 & 0,2 \\
4579 & II $4 \mathrm{p}^{2} \mathrm{~S}_{1 / 2}^{0} \rightarrow 4 \mathrm{~s}^{2} \mathrm{P}_{1 / 2}$ & 140 & 0,1 \\
3638 & III; $4 \mathrm{p}^{1} \mathrm{~F}_{3} \rightarrow 4 \mathrm{~s}^{1} \mathrm{D}_{2}^{0}$ & 450 & 1,0 \\
3511 & III; $4 \mathrm{p}^{3} \mathrm{P}_{2} \rightarrow 4 \mathrm{~s}^{3} \mathrm{~S}_{1}^{0}$ & 450 & 1,5 \\
2913 & IV; $4 \mathrm{p}^{2} \mathrm{D}_{5 / 2}^{0} \rightarrow 4 \mathrm{~s}^{2} \mathrm{P}_{3 / 2}$ & 1800 & 0,05 \\
\hline
\end{tabular}

Tab. 1. Schwellwertstrom und ausgekoppelte Spitzenleistung für die intensivsten Argon-Übergänge.

Der Entladekondensator war bei allen Linien $1 \mu \mathrm{F}$. Für jeden Übergang wurde der optimale Druck gewählt. Bei längerer Strompulsdauer $(40 \mu \mathrm{F})$ steigt die Leistung der Ar II-Übergänge noch um etwa eine Größenordnung (Abb. 4).
Zur Absoluteichung der ausgekoppelten Leistung wurde dasselbe Laserrohr als Gleichstrom-He-NeLaser betrieben, und der Multiplierstrom am Ausgang des Spektrographen mit dem Photostrom eines für $\lambda=6323 \AA$ geeichten Photoelementes verglichen. Bei Berücksichtigung der spektralen Empfindlichkeitskurve des Multipliers erhält man daraus die Leistung für alle Wellenlängen aus den gemessenen Ausgangsspannungen am Ableitwiderstand der Multiplieranode.

\section{Xenon}

Von den bei unseren experimentellen Bedingungen gefundenen 18 induzierten Xe-Übergängen wurden nur die 10 intensivsten näher untersucht. Wegen des großen Rohrdurchmessers von $7 \mathrm{~mm}$ spielt die Rekombination der Ionen an der Wand eine geringere Rolle, deshalb werden bevorzugt zweifach ionisierte Zustände angeregt, die wahrscheinlich durch einen Einstufenprozeß (wie beim Argon) von Termen des einfach ionisierten Atoms mit entsprechender Parität aus erreicht werden.

In Tab. 2 sind die untersuchten Linien mit den bisher veröffentlichten Termzuordnungen (BRIDGES ${ }^{1}$ ), den gemessenen Schwellwertströmen und ausgekoppelten Spitzenleistungen zusammengestellt. Auf optimale Auskopplung wurde kein Wert gelegt, deshalb sind die Leistungen im Resonator je nach Wellenlänge 200 - 500fach höher.

Da alle diese Linien auch bei Verwendung von Aluminiumspiegeln auftraten, muß der Gewinn der induzierten Emission größer als 10\% pro Meter sein.

\begin{tabular}{|c|c|c|c|}
\hline $\begin{array}{l}\text { Wellen- } \\
\text { länge } \\
(\AA)\end{array}$ & $\begin{array}{l}\text { Ionisationsgrad } \\
\text { und Utbergang } \\
\text { (nach BRIDGES) }\end{array}$ & $\begin{array}{c}\text { Minimaler } \\
\text { Schwell- } \\
\text { wert- } \\
\text { strom } \\
\text { (Ampere) }\end{array}$ & $\begin{array}{l}\text { Ausge- } \\
\text { koppelte } \\
\text { Spitzen- } \\
\text { leistung } \\
\text { (Watt) }\end{array}$ \\
\hline 5971 & $\mathrm{II} ; 6 \mathrm{p}^{2} \mathrm{P}_{3 / 2}^{0} \rightarrow 6 \mathrm{~s}^{2} \mathrm{D}_{3 / 2}$ & 540 & 2,5 \\
\hline 5394 & III; & 660 & 10 \\
\hline 5352 & III; & 480 & 20 \\
\hline 5259,9 & $\left.\mathrm{II} ; 7 \mathrm{~s}^{4} \mathrm{P}_{5 / 2} \rightarrow 6 \mathrm{p}^{4} \mathrm{D}_{5 / 2}^{0}\right)$ & & \\
\hline 5260,4 & $\mathrm{II} ; 6 \mathrm{p}^{2} \mathrm{P}_{3 / 2}^{0} \rightarrow 6 \mathrm{~s}^{2} \mathrm{D}_{3 / 2}$ & 540 & 25 \\
\hline 5261,9 & $\mathrm{II} ; 6 \mathrm{p}^{2} \mathrm{D}_{3 / 2}^{0} \rightarrow 6 \mathrm{~s}^{2} \mathrm{D}_{3 / 2}$ & & \\
\hline 5159 & III; & 730 & 1,5 \\
\hline 5007 & III; & 500 & 8 \\
\hline 4954 & III; & 400 & 25 \\
\hline 3645 & III; & 680 & 2,5 \\
\hline 3454 & III $; 6 \mathrm{p}^{1} \mathrm{D}_{2} \rightarrow 6 \mathrm{~s}^{1} \mathrm{D}_{2}^{0}$ & 1000 & 0,05 \\
\hline 3330 & IV?; (wahrscheinlich III) & 750 & 0,3 \\
\hline
\end{tabular}

Tab. 2. Schwellwertstrom und ausgekoppelte induzierte Spitzenleistung für die intensivsten Xe-Linien. 
Die drei Linien bei $5260 \AA$ konnten nur bei Messungen mit der Photoplatte getrennt werden. Bei Bestimmungen der Ausgangsleistung mit dem Multiphier wurden sie alle zusammen gemessen.

Obwohl die Schwellwertströme durchweg höher liegen als beim Argon, erreicht man größere Spitzenleistungen ${ }^{13}$. Anders als beim Argon unterscheiden sich die einfach- und zweifach-ionisierten Übergänge kaum im Absolutwert ihres minimalen Schwellwerts, aber auffällig ist dessen verschiedene Druckabhängigkeit (Abb. 6), die auch folgende Beobachtung erklärt: Vermindert man bei Stromstärken von etwa $1000 \AA ̊$ den Gasdruck, so sieht man bei Verwendung breitbandiger Resonatorspiegel deutlich eine entsprechende Farbänderung der induzierten Emission von orange über gelb nach grün bis blau.

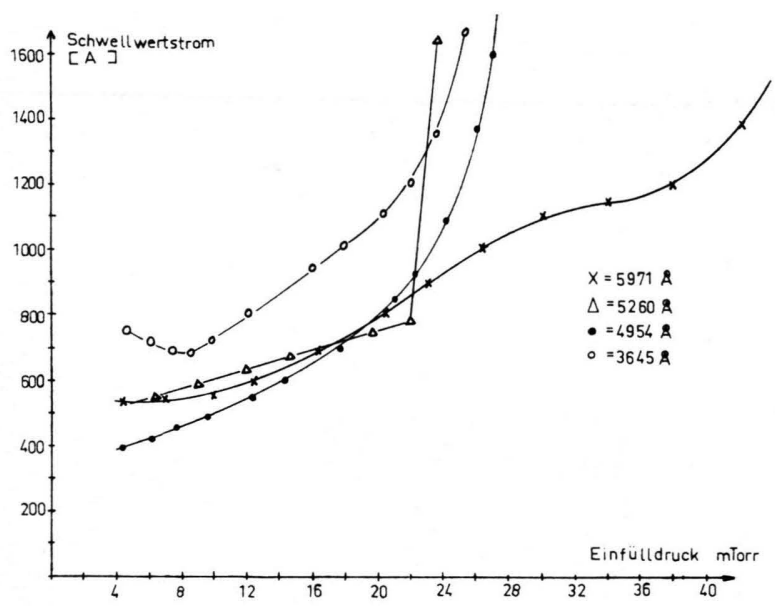

Abb. 6. Druckabhängigkeit des Schwellwertstromes für verschiedene induzierte Übergänge in Xenon.

Bei einigen Übergängen der einfach ionisierten Atome (z. B. $\lambda=5260 \AA$ ) steigt der Schwellwertstrom linear mit dem Druck an bis zu einem Maximaldruck, bei dem die induzierte Emission aufhört. Der Strombereich, in dem Inversion erreicht werden kann, nimmt dabei mit wachsendem Druck ab, ähnlich wie bei der Ar II-Linie in Abb. 2. Bei sehr hohen Strömen tritt dann aber wieder induzierte Emission auf, was darauf hinweist, daß ein neuer Anregungsmechanismus auftritt (siehe unten).

Bei der Untersuchung des zeitlichen Verlaufs der induzierten Emission zeigt sich ein komplizierteres Bild als beim Argon. Man kann etwa folgende Typen unterscheiden (Abb. $7 \mathrm{a}-\mathrm{d})$ :

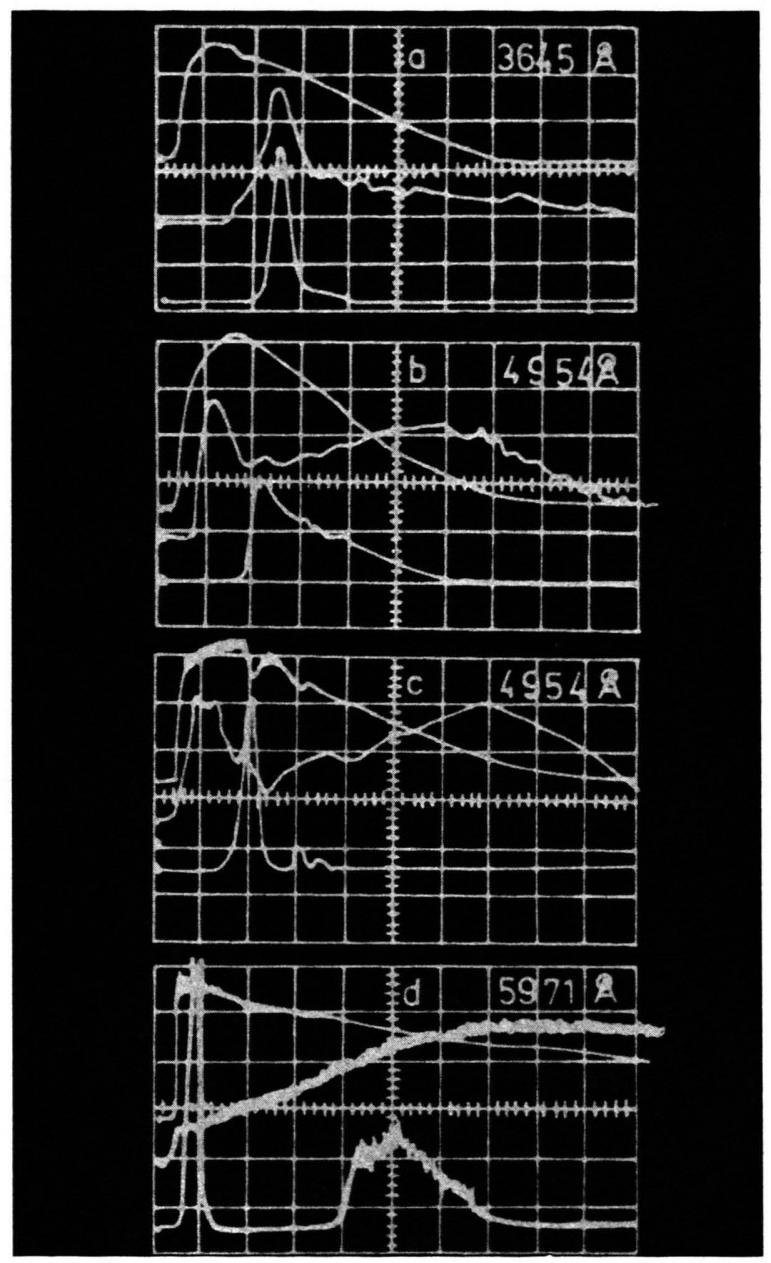

Abb. 7. Beispiele für verschiedene Erscheinungsformen der induzierten Emission bei einigen Xe-Übergängen. Zeit: $a-c$ : $1 \mu \mathrm{s} / \mathrm{cm}, 1 \mu \mathrm{F}, \mathrm{d}: 10 \mu \mathrm{s} / \mathrm{cm}, 40 \mu \mathrm{F}$.

a) Nur kurzzeitig erreichbare Inversion, die mit dem Maximum der spontanen Emission zusammenfällt. Die Verzögerungszeit zwischen Strom und induzierter Emission ist etwa $2 \mu \mathrm{s}$.

b) Die spontane Emission hat ein steiles Maximum zu Beginn des Strompulses und ein zweites breiteres, aber flacheres während des Abklingens der Entladung.

Induzierte Emission setzt nach diesem ersten Maximum ein, hat einen flachen Abfall, auf dem bei mittleren Drucken auch noch ein zweiter Puls erscheinen kann. Bei Vergrößerung der Entladungsdauer wurde die induzierte Emission nicht länger, so daß kontin- 
nuierlicher Betrieb auch für diesen Typ nicht zu erwarten ist.

c) Bei höheren Entladungsströmen wird der induzierte Puls plötzlich schmaler und höher, gleichzeitig erscheinen im Stromverlauf ausgeprägte Oszillationen, die zeitlich mit der induzierten Emission zusammenfallen.

Wegen der großen Stromdichte (bis $6000 \mathrm{~A} / \mathrm{cm}^{2}$ ) tritt hier wahrscheinlich Pincheffekt auf, der den Strom auf die Rohrachse hin komprimiert und den Entladungswiderstand kurzzeitig erhöht. Durch diese radiale Kompression des Plasmas scheint Inversion erzeugt zu werden.

BeNNET ${ }^{14}$ hat an gepulsten Ar II-Übergängen ähnliche Beobachtungen gemacht. In unserer Anordnung ist die Korrelation zwischen Laserpuls und Stromoszillation bei den zwei- und dreifach ionisierten Übergängen aber viel ausgeprägter als bei den einfach ionisierten. Oberhalb eines Minimalstromes trägt dieser Anregungsmechanismus bei allen diesen Übergängen wesentlich zur induzierten Leistung bei. Bei $\lambda=3645 \AA$ z. B. liegt der Entladungsstrom in Abb. 7 a dicht unter diesem Minimalwert. Bei weiterer Erhöhung der Stromstärke erscheint vor dem abgebildeten Laserpuls ein neuer, der zeitlich mit dem bereits in Abb. $7 \mathrm{a}$ angedeuteten Stromminimum zusammenfällt und bald die 20 -fache Intensität des zweiten Pulses erreicht.

d) Bei den Linien des einfach ionisierten Xenon erscheint nach dem kurzen, intensiven induzierten Puls ein zweiter, um eine Größenordnung schwächerer, dessen Dauer aber mit wachsender Entladungsdauer zunimmt, und dessen Anregungsmechanismus bei geeigneten Bedingungen zu kontinuierlicher Inversion führen kann.

$\mathrm{Da}$ in $\mathrm{Abb} .7 \mathrm{~d}$ die induzierte Emission wieder aufhört, obwohl die Besetzungszahl des oberen Niveaus zunimmt, muß das untere Niveau noch stärker be- völkert werden. Das liegt zum Teil auch an der Verwendung breitbandiger Resonatorspiegel, bei denen gleichzeitig mehrere induzierte Übergänge angeregt werden, die auf dasselbe untere Niveau führen.

Bei $\lambda=3645 \AA$ tritt, ähnlich wie beim Ar III, $3511 \AA$, im oberen Drurckbereich ein langer, schwacher Nachpuls auf $(10 \mathrm{~mW})$, der kontinuierliche Emission erwarten läßt.

\section{Zusammenfassung}

Die Untersuchung der induzierten Emission in gepulsten Ar- und Xe-Entladungen zeigt für die optimalen experimentellen Bedingungen folgendes Bild:

Für Übergänge der einfach ionisierten Atome sind Entladungsrohre mit kleinem Querschnitt günstiger, weil bei großem Rohrdurchmesser die Schwellwertströme hoch sind, und die Inversion und damit die erreichbare Leistung durch Reabsorption der Resonanzstrahlung begrenzt wird. Die höher ionisierten Übergänge werden dagegen bei größerem Rohrquerschnitt stärker angeregt und man erhält Spitzenleistungen im Resonator bis zu $6 \mathrm{~kW}$ für diese Übergänge bei Xenon. Optimale Einfülldrucke liegen bei Ar zwischen 10-20 mTorr, bei Xe zwischen 5-10 mTorr. Weitere Erniedrigung des Druckes vermindert zwar bei den meisten Linien den Schwellwert, aber auch die Pulsbreite der induzierten Emission und für viele Linien auch die maximal erreichbare Spitzenleistung. Diese wächst bei den Übergängen der höher ionisierten Atome viel stärker mit der Feldstärke als bei den einfach ionisierten, steigt aber mit dem Strom nur bis zu einem vom Druck abhängigen Sättigungswert.

Wir möchten Herrn Stamer für seine Mithilfe bei einigen Messungen und beim Aufbau der Apparatur danken.

14 W. R. Bennet JR., Appl. Opt., Suppl. on Chemical Lasers, S. 18 [1965]. 